\section{JTI}

JOURNAL OF

TRAUMA AND INJURY

\title{
An Irreducible Hip Dislocation with Femoral Head Fracture
}

\author{
Tae-Seong Kim, M.D. ${ }^{1,2}$, Chang-Wug Oh, M.D., Ph.D. ${ }^{1,2}$, \\ Joon-Woo Kim, M.D., Ph.D. ${ }^{1,2}$, Kyeong-Hyeon Park, M.D. ${ }^{1,2}$ \\ ${ }^{1}$ Regional Trauma Center, Kyungpook National University Hospital, Daegu, Korea \\ ${ }^{2}$ Department of Orthopaedic Surgery, School of Medicine, Kyungpook National University, \\ Daegu, Korea
}

Received: July 9, 2018

Revised: September 14, 2018

Accepted: September 22, 2018

\section{Correspondence to}

Chang-Wug Oh, M.D., Ph.D.

Department of Orthopaedic, Kyungpook National University Hospital, School of

Medicine, Kyungpook National University, 130 Dongdeok-ro, Jung-gu, Daegu 41944, Korea

Tel: + $82-53-420-5630$

Fax: +82-53-422-6605

E-mail:cwoh@knu.ac.kr
Urgent reduction is required in cases of traumatic hip dislocation to reduce the risk of avascular necrosis of the femoral head. However, in cases of femoral head fractures, the dislocated hip cannot be reduced easily, and in some cases, it can even be irreducible. This irreducibility may provoke further incidental iatrogenic fractures of the femoral neck. In an irreducible hip dislocation, without further attempting for closed reduction, an immediate open reduction is recommended. This can prevent iatrogenic femoral neck fracture or avascular necrosis of the femoral head, and save the natural hip joint.

Keywords: Hip dislocation; Closed fracture reduction; Femoral head fractur

\section{INTRODUCTION}

A fracture of the femoral head with hip dislocation is a rare and severe injury [1,2]. About $5-15 \%$ of posterior hip dislocations have been reported to be associated with femoral head fractures $[1,3,4]$. Early diagnosis and accurate reduction are necessary for the patients with femoral head fracture-dislocations [3,5]. A delayed reduction may result into an osteonecrosis of the femoral head later [4]. Therefore, an emergent reduction is preferred to reduce the risk of femoral head osteonecrosis $[4,6]$. Although rarely (about $9 \%$ of all hip dislocations), irreducible hip dislocations with femoral head fractures have been reported [7]. An increased risk of iatrogenic femoral neck fracture after a closed reduction as compared to those after routine femoral head fracture-dislocations $[8,9]$ suggests the need for an open reduction to avoid complications. This study describes two cases of irreducible hip dislocations with femoral head fractures.

This is an Open Access article distributed under the terms of the Creative Commons Attribution Non-Commercial License (http://creativecommons.org/licenses/by-nc/4.0/) which permits unrestricted noncommercial use, distribution, and reproduction in any medium, provided the original work is properly cited. 


\section{JTI}

\section{CASE REPORT}

\section{Case 1}

A 60-year-old male presented with an injury after a fall from 5 meters height. The patient had multiple organ damage, including intracerebral hemorrhage with basal skull fracture, cervical spine fracture, multiple rib fractures with hemo-pneumothorax, liver injury, and fracture-dislocation of the right femoral head with posterior wall fracture of the acetabulum-type IV per the Pipkin classification (Fig. 1). Considering the general condition of the patient, physiological stabilization was prioritized. After the initial resuscitation, a closed reduction for the right hip dislocation was attempted with the Allis method; but the dislocation was irreducible. After that, we performed a computed tomography (CT) scanning, which showed a locked femoral head at the posterior rim of the acetabulum (Fig. 2). The patient's vital signs were unstable (blood pressure, 80/40; heart rate, 120 ), and because of the brain and lung injury, we were unable to attempt an open reduction immediately. The patient was shifted to the intensive care unit (ICU). In the ICU, we attempted for a closed reduction once again, under full sedation with muscle relaxants (rocuronium bromide $50 \mathrm{mg}$ intravenous). We attempted for a closed reduction several times; but unfortunately, the neck of the femur was broken (Fig. $3)$. The patient subsequently developed multiorgan failure, which prevented a surgical intervention for the hip dislocation until 4 weeks after the injury. After the patient has recovered, we have to choose the treatment options. Open reduction and internal fixation was one of the treatment option, but 4 weeks have passed since the accident. There is high probability of nonunion, it means another surgery is needed, revision surgery for nonunion or even arthroplasty. The patient does not want further more surgery and treated with a total hip arthroplasty (Fig. 4).
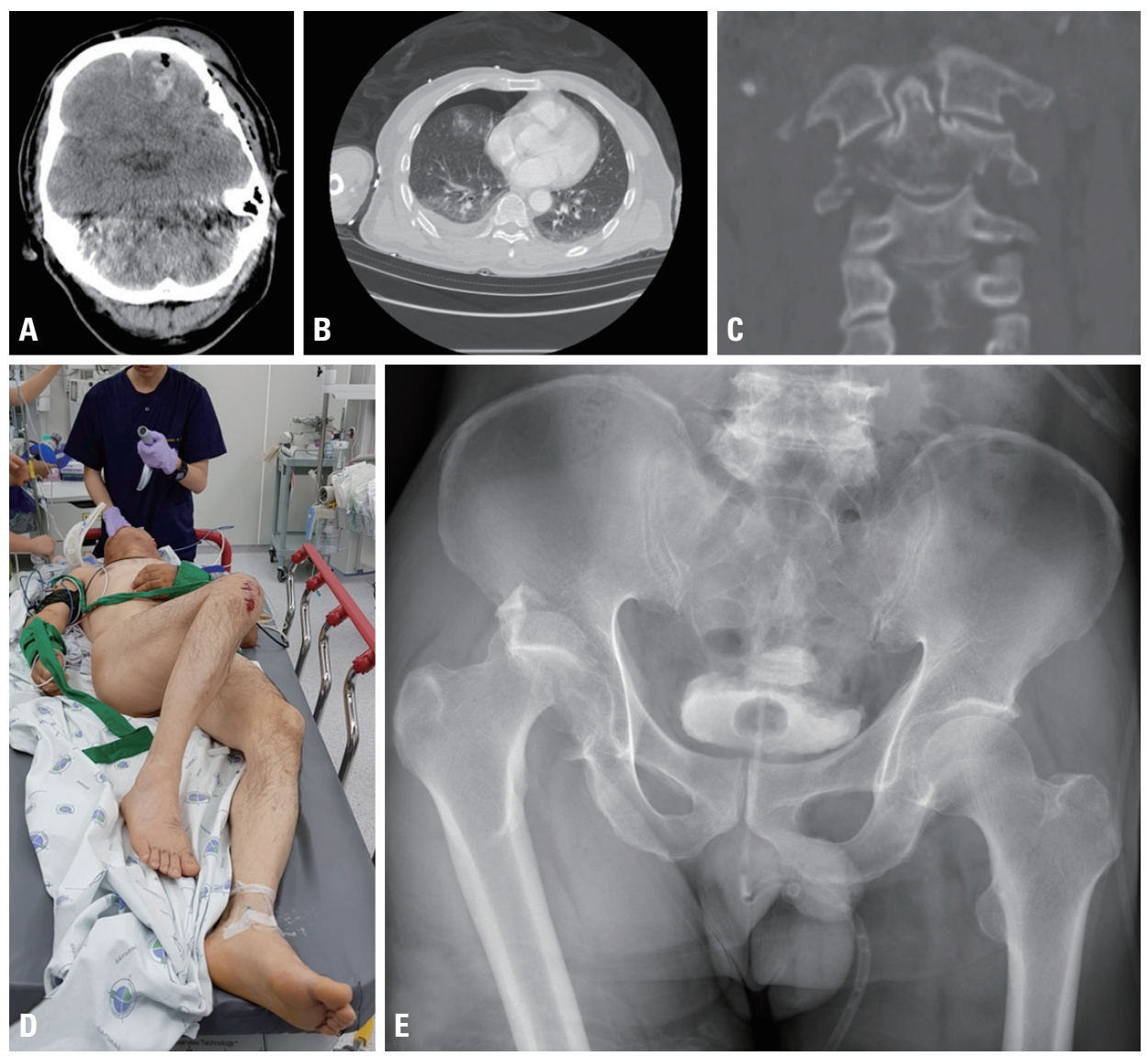

Fig. 1. The patient had multiple organ damages. (A) Intracerebral hemorrhage. (B) Right hemo-pneumothorax. (C) Cervical spine fracture. (D) Right hip showed flexion, internal rotation, adduction, just like the typical appearance of hip dislocation. (E) An X-ray of the pelvis (anterior posterior view) showing fracture-dislocation of the right hip joint. 

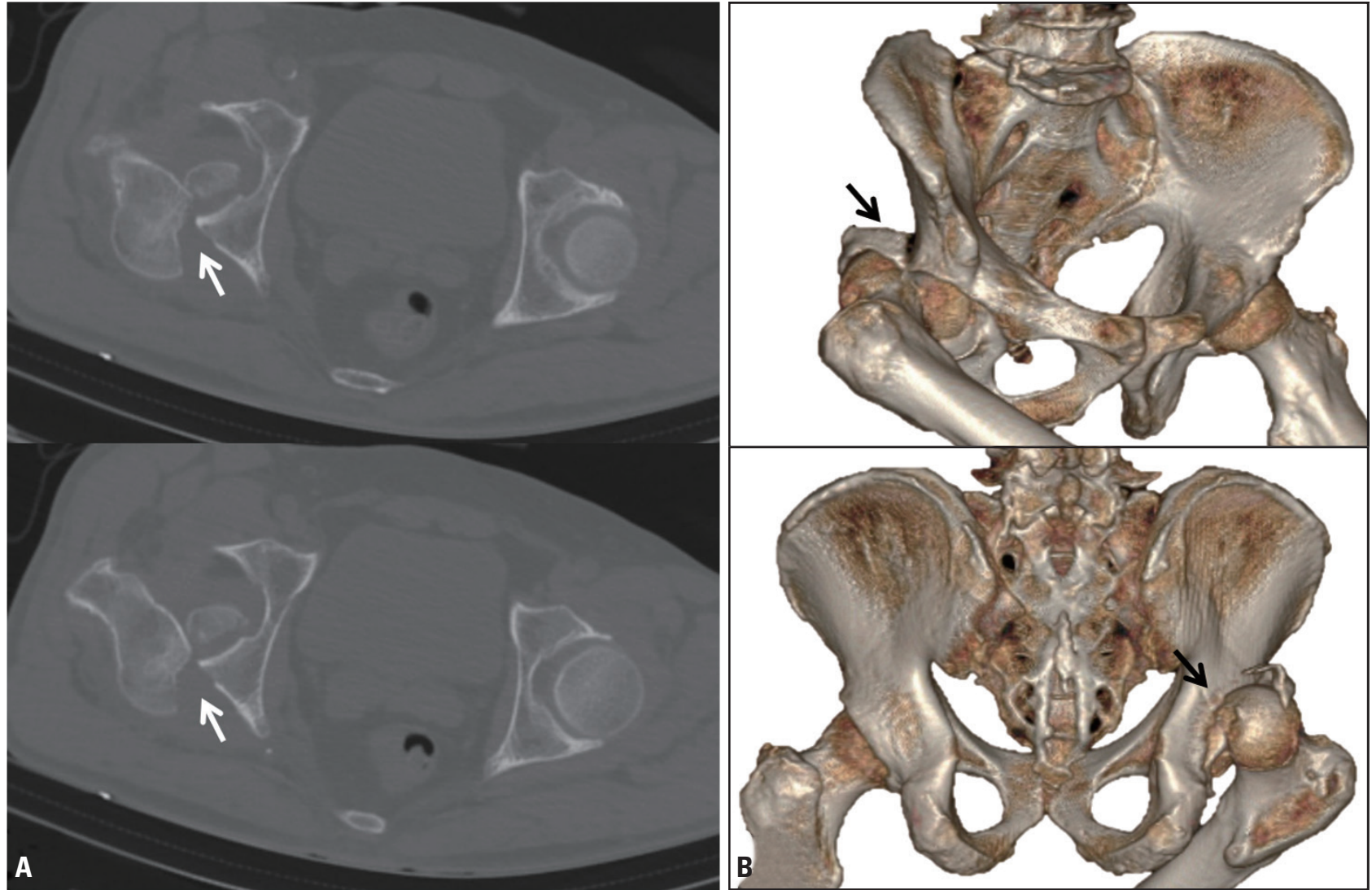

Fig. 2. (A) CT scans of the pelvis showing fracture-dislocation of the right hip joint. Femoral head was seen to be locked at the posterior rim of the acetabulum (with arrows). (B) A 3D reconstruction image of the CT scan showing the locked femoral head with the fracture of the posterior wall of the acetabulum (black arrows indicates that locked femoral head). CT: computed tomography.
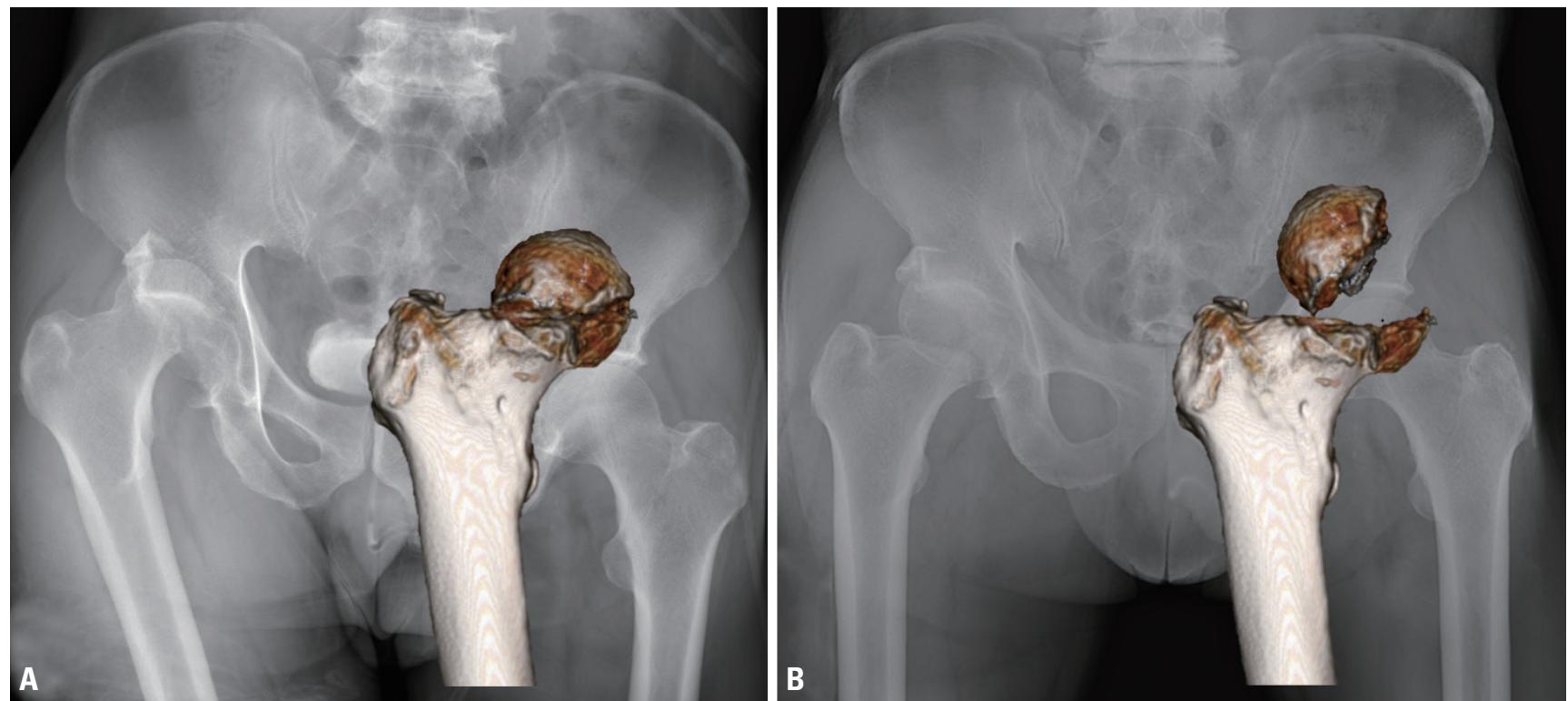

Fig. 3. (A) An initial X-ray of the pelvis (anterior posterior view) and 3D reconstruction image of the right femur. (B) After the failure of the closed reduction of the dislocation, the femoral neck fractured. 


\section{JTI}

\section{Case 2}

A 31-year-old male was injured in a high-speed car accident. The patient was transferred by a helicopter to our polytrauma unit, where the patient reported pain in the left hip, left knee joint. The patient was conscious, and his medical interview provided the information about the mechanism of the injury-a dashboard injury. The phys-

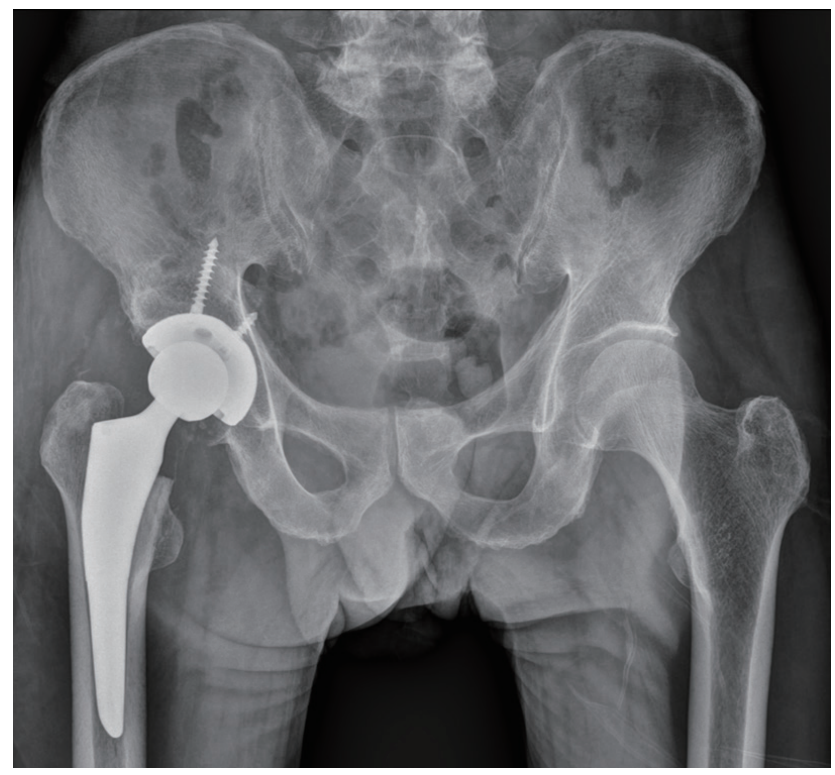

Fig. 4. Four weeks after the injury, a total hip arthroplasty was performed. ical examination of the left hip revealed pain, shortened left lower limb, compulsory flexion, adduction and slight internal rotation of the left hip, limited range of motion, and deformation of the left gluteal area. External wound and tenderness of the left knee joint were also noted (Fig. 5). No additional injuries to other parts were found during the examination. Nevertheless, the patient exhibited left side sciatic nerve palsy. He could not dorsiflex the left ankle joint, and experienced a tingling sensation in the whole left leg. Subsequently, a radiological evaluation was performed. An antero-posterior X-ray of the pelvis showed a posterior dislocation of the left hip; the image of the posterior rim of the acetabulum was unclear. A closed reduction of the dislocated hip was attempted very gently; but failed, because it seemed like an irreducible femoral head fracture dislocation. Therefore, we performed CT scanning, which revealed a femoral head fracture-type III per the Pipkin classification, and a posterior hip dislocation; about $30 \%$ of the femoral head was seen to be fragmented and located in the acetabulum. Rest of the femoral head was dislocated in the posterior direction. In addition, we found an incomplete femoral neck fracture (Fig. 6). The rim of the posterosuperior acetabulum was fractured, but the fragment was very small. Lateral and antero-posterior X-rays of the left knee joint showed comminuted, articular fracture of the patella. The patient
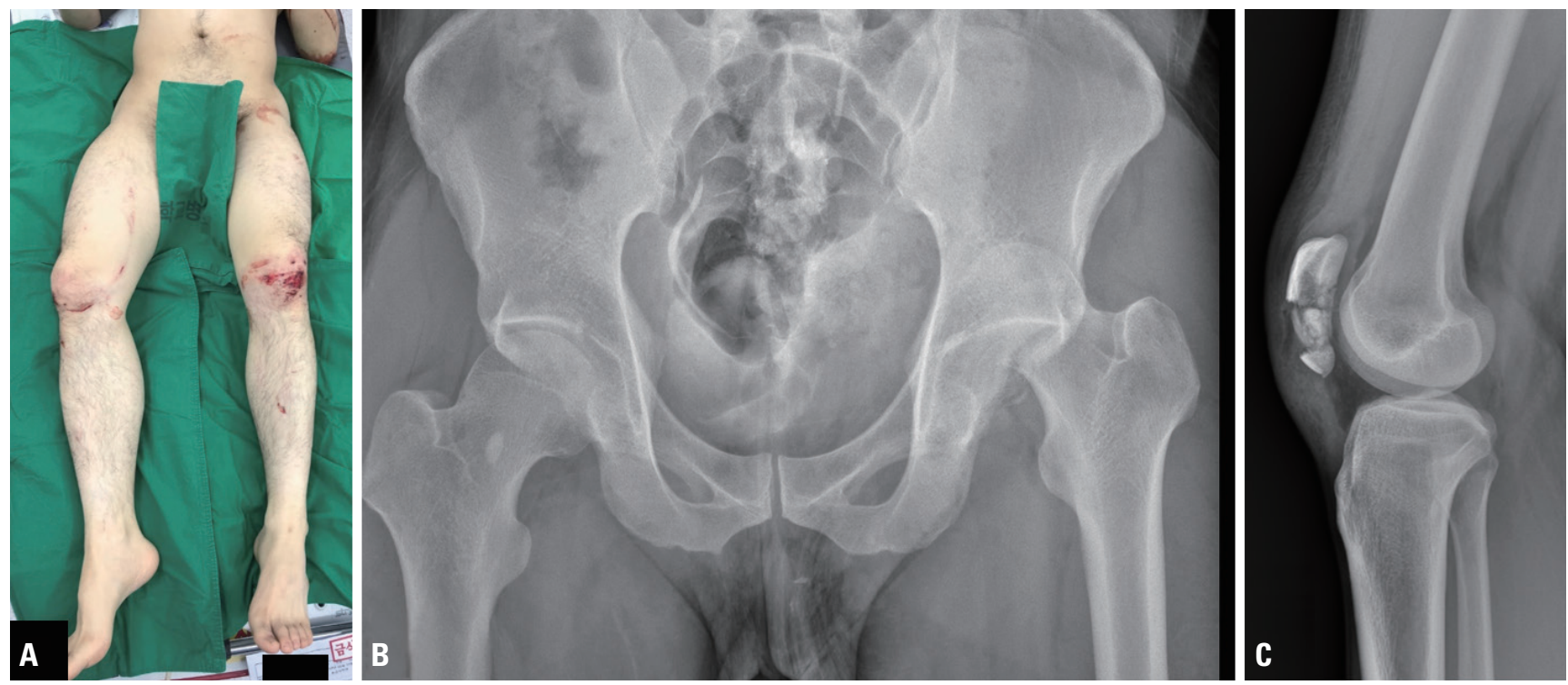

Fig. 5. (A) A pre-operative X-ray of the pelvis anterior posterior view showing fracture dislocation of the left hip joint. (B) The patient had an ipsilateral knee injury and slightly internally rotated hip. (C) An X-ray of the left knee joint (lateral view) showing comminuted patella fracture. 


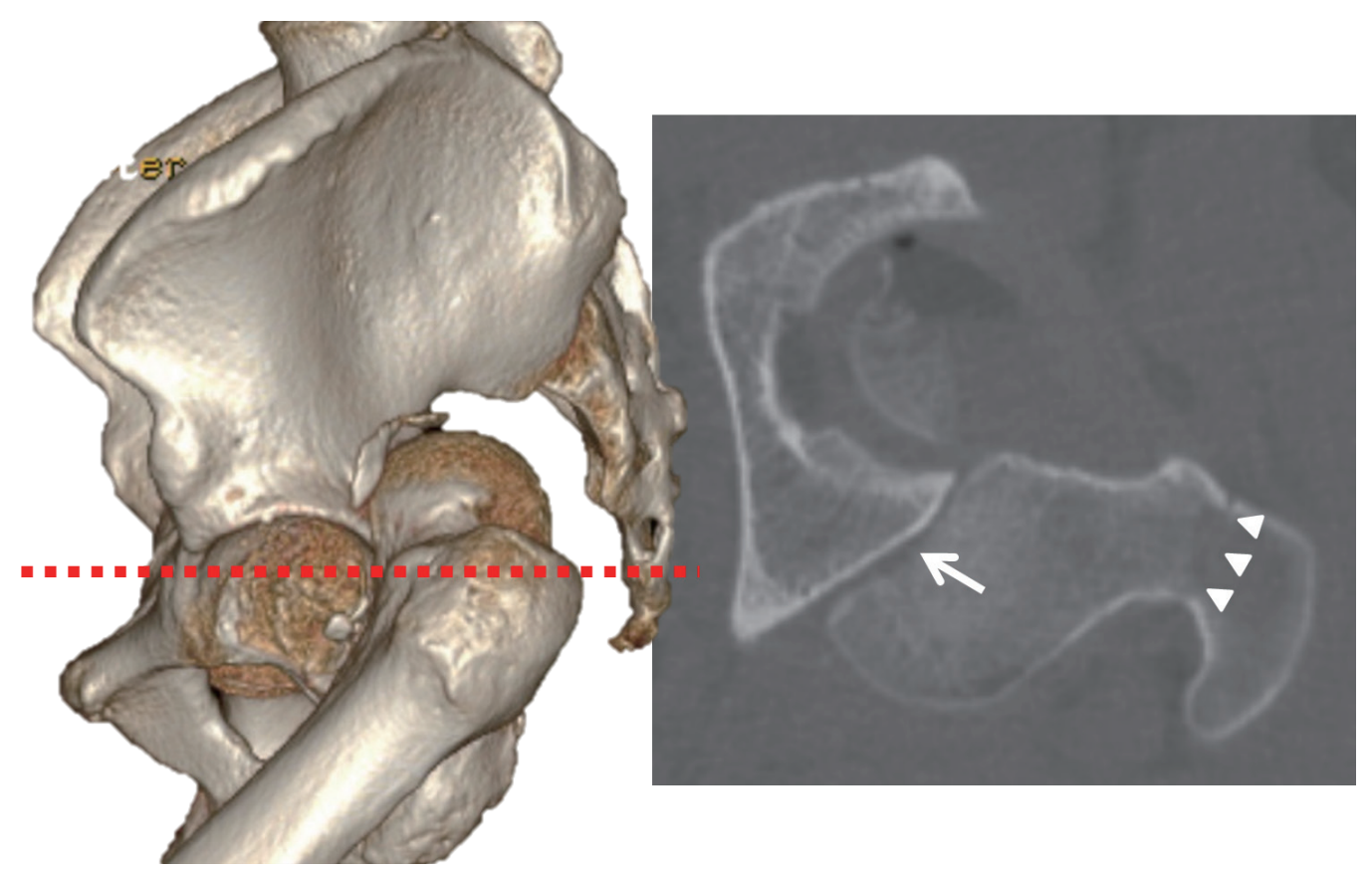

Fig. 6. Pre-operative axial CT scans of the pelvis showing fracture-dislocation of the left hip joint. An axial CT scan of the pelvis showing (dotted line in the 3D image indicates the level of the axial cut). The femoral head was seen to be locked at the posterior rim of the acetabulum (with arrow), and the fragments of the femoral head were seen inside the acetabulum. The white triangles indicate an incomplete femoral neck fracture. CT: computed tomography.
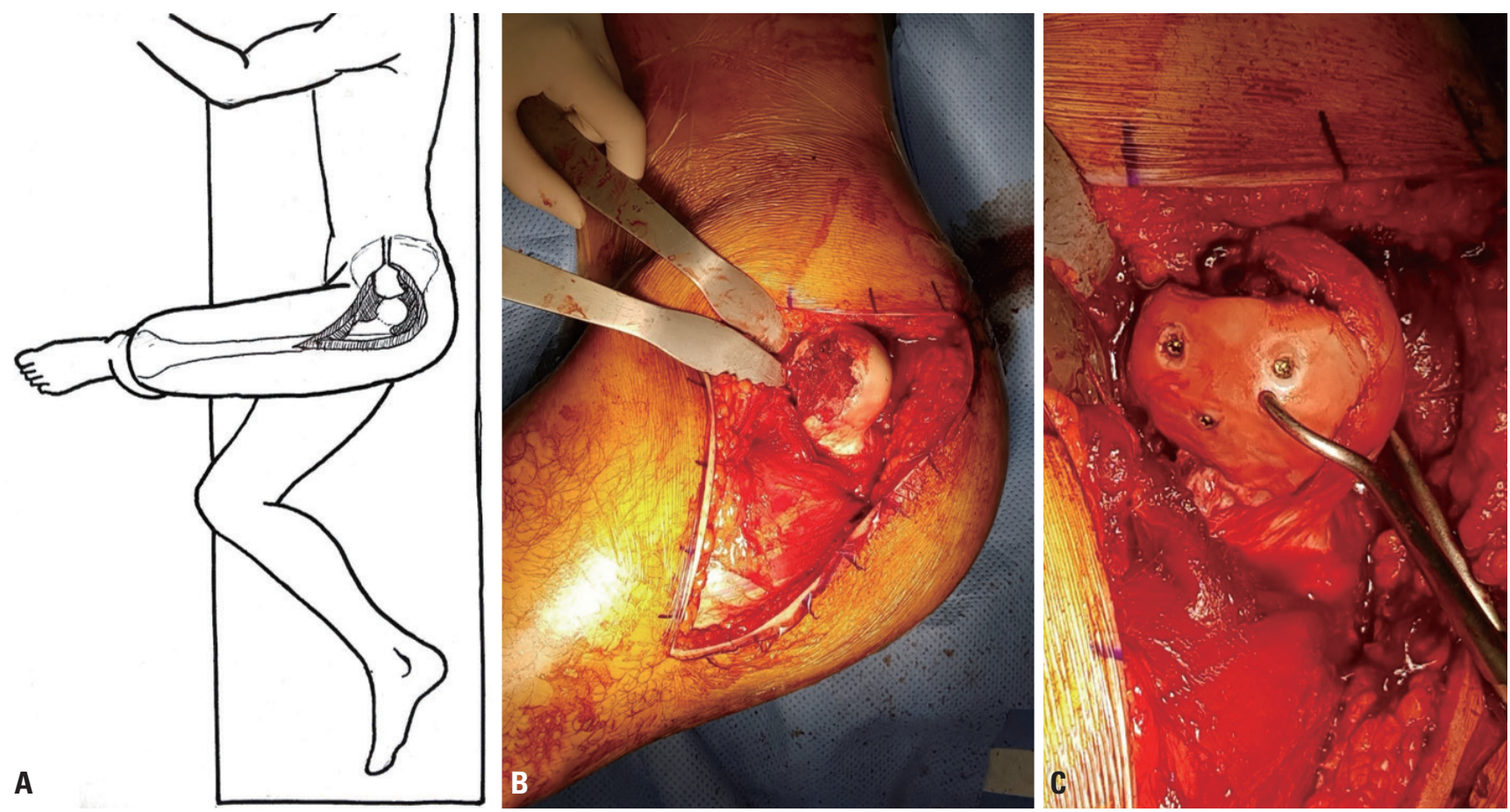

Fig. 7. (A) The patient in the lateral position for the surgical procedure in the Kocher-Langenbeck posterior approach. (B) After the surgical reduction of the dislocated hip by the trochanteric osteotomy, we had good visualization of the surface plane of the fractured femoral head. (C) The femoral head fracture fragments were reduced under direct observation, fixed with 3 cortical screws, and buried beneath the cartilage level. 


\section{JTI}

was referred to the Orthopedic Department. We selected the Kocher-Langenbeck posterior approach and performed a surgical reduction of the dislocated hip through trochanteric osteotomy. The femoral head fracture fragments in the acetabulum were removed; and then, reduced under direct observation, temporary fixed with the reduction forceps, stabilized with 3 cortical screws (diameter $2.7 \mathrm{~mm}$ ), and buried beneath the cartilage level (Fig. 7). Subsequently, the hip joint was relocated. At the same time, we also checked the sciatic nerve, which was intact. The femoral neck fracture was fixed in situ with three cannulated screws (diameter $6.5 \mathrm{~mm}$ along with the washer). The posterior wall fragment was too small to fix, and it was not found to affect hip joint stability; hence, we removed the fragment only. The joint-capsule and soft tissues were sutured. The patella fracture was treated with the mid-axial longitudinal approach and tension band wiring. After the surgery, we took a postoperative X-ray of the hip and knee joints to assess the quality of reduction; and furthermore, performed CT scanning of the affected hip to gain a detailed insight (Fig. 8). After 3 months, sciatic nerve neuropraxia was recovered, with active dorsiflexion of the ankle. Unlike the case 1, after diagnosing the irreducibility, in this patient an immediate open reduction was performed without further attempting for the closed reduction, which saved the natural hip joint and also prevented from iatrogenic femoral neck fracture.

\section{DISCUSSION}

The first report of the femoral head fracture-dislocation was presented by Birkett [10] in 1869 after an autopsy of a victim of fall. In 1957, Pipkin reviewed a series of 25 cases, and proposed a classification of femoral head fractures associated with posterior dislocation of the hip $[1,11]$. The mechanism of injury in the majority of cases includes that from a dashboard, in which the seat of the occupant is not protected by a seat belt $[1,11,12]$. It is caused when a force is applied parallel to the axis of the femur in a proximal direction with the hip flexed in varying proportions. If the hip is flexed at less than $60^{\circ}$, the femoral head may impinge against the firm posterior-superior rim, and a fracture of the femoral head may occur $[1,11]$. These frac-
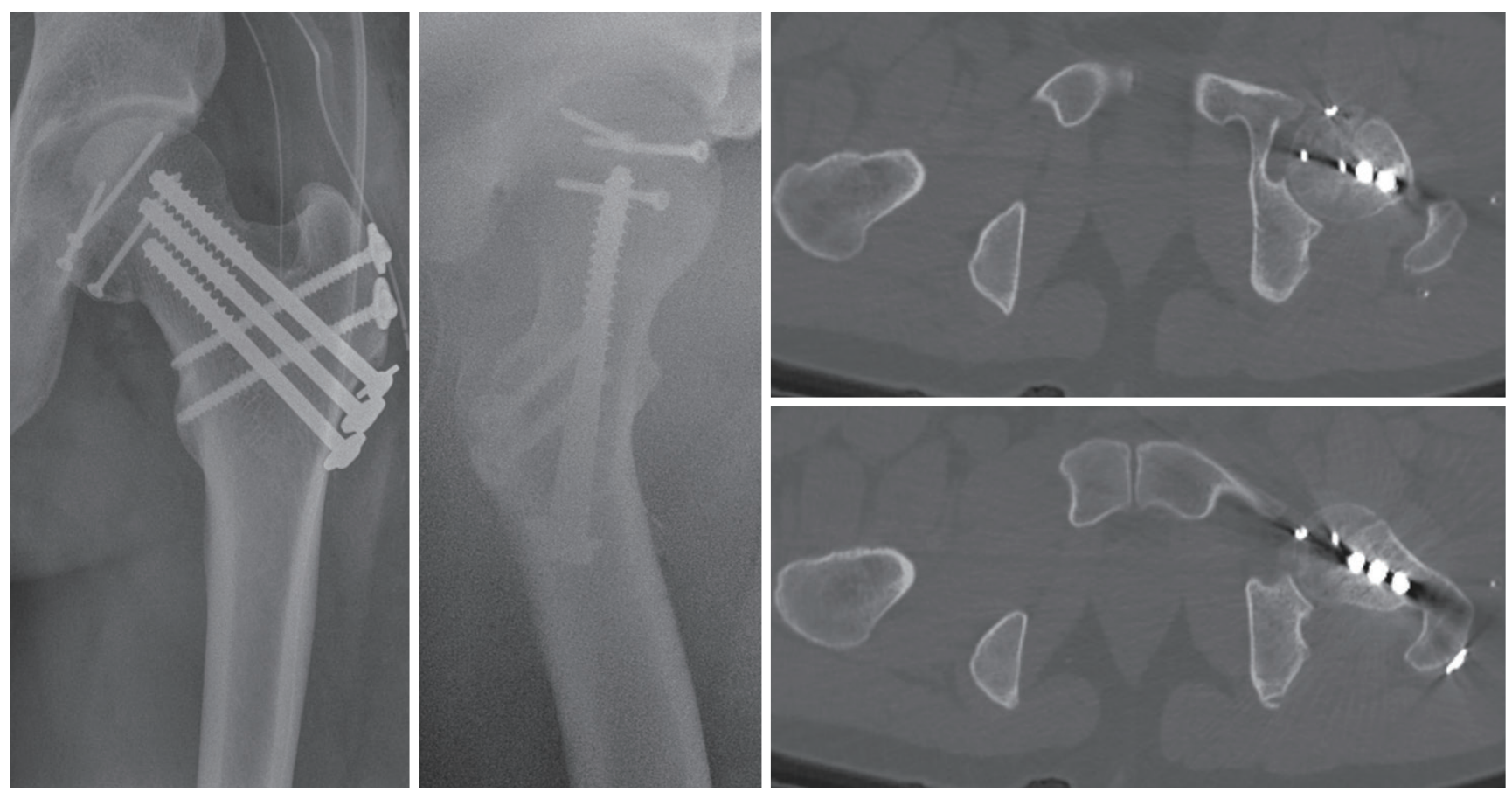

Fig. 8. A post-operative X-ray and an axial CT scan of the pelvis showing anatomic reduction and stabilization of the fracture. The posterior wall fragment was too small to fix, and it was not found to affect hip joint stability, so we removed the fragment. CT: computed tomography. 
tures require early closed reduction to protect the sciatic nerve, decrease pressure on the femoral head articular cartilage, and to prevent femoral head osteonecrosis. A closed reduction of this injury may initially be unsuccessful in certain cases, usually because of the intra-articular fragments that may block the reduction. Lawrence et al. [13] reported a rare case of an irreducible femoral head fracture-dislocation, which showed the broken femoral head stuck on the posterior wall of the acetabulum. The immobility or locking of the hip area is necessary to be identified as soon as possible. First, the clinical presentation of an irreducible hip joint injury is defined as slight but fixed flexion, immobile but neutrally rotated, and with obvious shortening of the injured side. However, a typical posterior hip dislocation shows a flexed, internally rotated, and adducted leg $[7,12]$. Therefore, the rotation of an injured leg may be variable, and may be affected by the surface plane of the fractured femoral head component and the direction of its engagement at the posterior acetabulum (Fig. 9) [14]. Second, the imaging studies of a hip dislocation with femoral head fractures is necessary for diagnosing cases of irreducibility. The radiographic examination of the antero-posterior projection of the injured hip showed a portion of the femoral head retained in the acetabulum, with the remaining femoral head components, posterior and superior, were found to be intact relative to the acetabulum. The screening in an obturator foramen view revealed a specific finding of the engage-
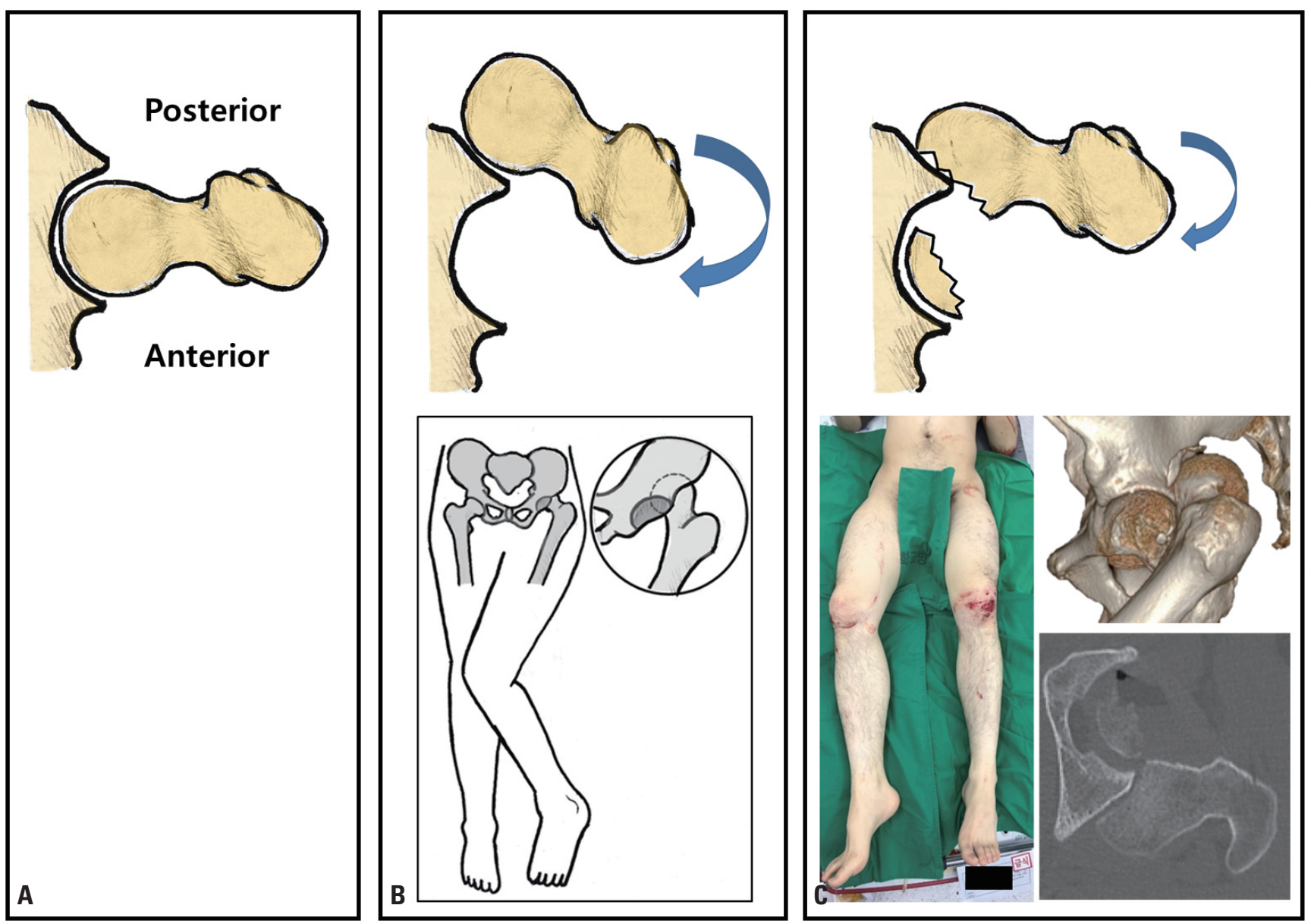

Fig. 9. (A) The top view of the hip joint. (B) In most cases, the posterior hip dislocation shows a flexed, internally rotated, and adducted leg because of the impingement between the femur and acetabulum (arrow indicates more internal rotation than C). (C) The femoral head fracture dislocation is less internally rotated, affected by the surface plane of the fractured femoral head; or it can be any other directions, which depends on the direction of its engagement at the posterior acetabulum (arrow indicates less internal rotation than B). 
ment or locking of the femoral head component against the posterior rim of the acetabulum. Moreover, CT scanning is commonly performed on the patients who present with severe or high-energy trauma. Most of these patients initially undergo CT scanning. The coronal or sagittal CT images of our patient revealed a fractured surface of the femoral head component locked on the posterior rim of the acetabulum. Three-dimensionally reconstructed CT images showed that the remaining femoral head segment with the femoral head component locked against the posterior rim of the acetabulum would be at a high risk of accidental femoral neck fractures when attempting a closed reduction. Based on these findings, as well as on clinical correlations, we recommend an open reduction, without further attempts for the closed reduction, in an irreducible femoral head fracture-dislocation. In case 1 arthroplasty was performed after iatrogenic femoral neck fracture and in case 2 open reduction and internal fixation was performed. There are limitations in determining the prognosis of open reduction and internal fixation and comparing side effects (avascular necrosis of hip etc.) with the two cases. However, it is adequate as a case report in that the case with femoral head fracture dislocation suggests caution is advised during reduction when locking is present between the femoral head and the posterior wall of acetabulum. Also, we cannot exclude that the linear femoral neck fracture in case 2 is an iatrogenic fracture during the first attempt of reduction. Therefore, when femoral head fracture dislocation is present in the X-ray, it is advantageous to determine the presence of locking between the femoral head and the acetabulum with a CT scan before performing reduction.

Femoral head fractures with hip dislocation need an emergent orthopedic surgery and an optimal reduction within 6 hours post-trauma; otherwise, they may result into an increased risk of avascular necrosis of the femoral head. Repeated or forceful closed reduction of irreducible femoral head fracture-dislocation injuries may result in iatrogenic femoral neck fractures. Before attempting a reduction, careful examination with plain radiographs, even CT scans may be helpful for determining the safest treatment option. Once an irreducible femoral head fracture-dislocation is identified, an orthopedic management should be initiated as soon as possible; and an immediate open reduction should be performed.

\section{REFERENCES}

1. Stannard JP, Harris HW, Volgas DA, Alonso JE. Functional outcome of patients with femoral head fractures associated with hip dislocations. Clin Orthop Relat Res 2000;377:44-56.

2. Marchetti ME, Steinberg GG, Coumas JM. Intermediate-term experience of Pipkin fracture-dislocations of the hip. J Orthop Trauma 1996;10:455-61.

3. Yang RS, Tsuang YH, Hang YS, Liu TK. Traumatic dislocation of the hip. Clin Orthop Relat Res 1991;265:218-27.

4. McKee MD, Garay ME, Schemitsch EH, Kreder HJ, Stephen DJ. Irreducible fracture-dislocation of the hip: a severe injury with a poor prognosis. J Orthop Trauma 1998;12:223-9.

5. Pietrafesa CA, Hoffman JR. Traumatic dislocation of the hip. JAMA 1983;249:3342-6.

6. Hougaard K, Thomsen PB. Traumatic posterior dislocation of the hip--prognostic factors influencing the incidence of avascular necrosis of the femoral head. Arch Orthop Trauma Surg 1986;106:32-5.

7. Mehta S, Routt ML Jr. Irreducible fracture-dislocations of the femoral head without posterior wall acetabular fractures. J Orthop Trauma 2008;22:686-92.

8. Pipkin G. Treatment of grade IV fracture-dislocation of the hip. J Bone Joint Surg Am 1957;39-A:1027-42 passim.

9. Epstein HC. Posterior fracture-dislocations of the hip; longterm follow-up. J Bone Joint Surg Am 1974;56:1103-27.

10. Birkett J. Traumatic dislocation of the head of the femur complicated with its fracture. Med Chir Trans 1869;51:133-8.

11. Lang-Stevenson A, Getty CJ. The Pipkin fracture-dislocation of the hip. Injury 1987;18:264-9.

12. Henle P, Kloen P, Siebenrock KA. Femoral head injuries: which treatment strategy can be recommended? Injury 2007;38:47888.

13. Lawrence DA, Smitaman E, Baumgartner M, Haims A. A rare but radiographically recognizable cause of an irreducible hip fracture-dislocation. Clin Imaging 2013;37:595-7.

14. Park KH, Kim JW, Oh CW, Kim JW, Oh JK, Kyung HS. A treatment strategy to avoid iatrogenic Pipkin type III femoral head fracture-dislocations. Arch Orthop Trauma Surg 2016;136:110713. 